\title{
Untargeted Metabolomics Based on GC-MS and Chemometrics: A New Tool for the Early Diagnosis of Strawberry Anthracnose Caused by Colletotrichum theobromicola
}

\author{
Tan Dai, Xunian Chang, Zhihong Hu, Li Liang, Mingyou Sun, Pengfei Liu, ${ }^{\dagger}$ and Xili Liu \\ Department of Plant Pathology and MOA Key Lab of Pest Monitoring and Green Management, College of Plant Protection, \\ China Agricultural University, Beijing 100193, China
}

\begin{abstract}
To prevent the spread of anthracnose in strawberry plants and characterize the metabolic changes occurring during plant-pathogen interactions, we developed a method for the early diagnosis of disease based on an analysis of the metabolome by gas chromatography-mass spectrometry. An examination of the metabolic profile revealed 189 and 202 total ion chromatogram peaks for the control and inoculated plants, respectively. A partial least squares discriminant analysis (PLS-DA) model was conducted for the reliable and accurate discrimination between healthy and diseased strawberry plants, even in the absence of disease symptoms (e.g., early

galactose, gallic acid, malic acid, methyl $\alpha$-galactopyranoside, phosphate, and shikimic acid). At least some of these potential biomarkers may be applicable for the early diagnosis of anthracnose in strawberry plants. Moreover, these metabolites may be useful for characterizing pathogen infections and plant defense responses. This study confirms the utility of metabolomics research for developing diagnostic tools and clarifying the mechanism underlying plant-pathogen interactions. Furthermore, the data presented herein may be relevant for developing new methods for preventing anthracnose in strawberry seedlings cultivated under field conditions.
\end{abstract} stages of infection). ANOVA (analysis of variance) and orthogonal partial least squares analysis (OPLS) identified 20 metabolites as tentative biomarkers of Colletotrichum theobromicola infection (e.g., citric acid, D-xylose, erythrose,

Keywords: Colletotrichum theobromicola, strawberry anthracnose, disease diagnosis, metabolomics, chemometrics

Strawberry (Fragaria $\times$ ananassa), belonging to the family Rosaceae, is a hybrid fruit species cultivated worldwide. Since the 1980s, the strawberry industry in China has expanded rapidly and currently represents a significant part of the fruit industry (Gareau 2008). Globally, China is the top strawberry-producing country (Simpson 2018). Strawberry plants are mainly cultivated in greenhouses, open fields, as well as large and small plastic sheds. The continuous cultivation of strawberry plants over several years has resulted in the development of serious diseases, including anthracnose, which has become one of the major diseases threatening strawberry production in China and worldwide. Anthracnose is becoming more problematic for strawberry growers, partly because of the increasing popularity of industrialized seedling production. Anthracnose was detected in strawberry plants as early as 1931, which is when Brooks observed infected stolons and petioles of strawberry plants grown in Florida (Brooks 1931). A previous study determined that similar strawberry anthracnose symptoms affecting all plant parts were induced by two Colletotrichum species complexes (Buddie et al. 1999). Crown symptoms and fruit rot would be formed after the appearance of reddish-brown necrotic areas, from pathogen infection (Ureña-Padilla et al. 2002). In some cases, root necrosis has been associated with stunting as well as the wilting and eventual death of plants (Freeman et al. 2001). After infecting strawberry plants, the pathogen normally enters an incubation period, during which symptoms are not visible, and may be transmitted when seedlings are transplanted. Suitable temperatures and

${ }^{\dagger}$ Corresponding author: P. Liu; pengfeiliu@cau.edu.cn

Funding: This study was supported by the National Natural Science Foundation of China (U160310041), the Special Fund of the National Science Foundation of China (31772192), and the National Key Research and Development Program of China (2017YFD0201602).

The author(s) declare no conflict of interest.

Accepted for publication 9 May 2019.

(C) 2019 The American Phytopathological Society humidity may result in serious outbreaks of strawberry anthracnose. Additionally, latent pathogens can adversely affect disease diagnoses, such as when fungal biomass is too low to be detected using normal methods (Israel et al. 1973; Sewell 1979).

Metabolomics is one of the most recently introduced (Fiehn et al. 2000; Roessner et al. 2000), and fastest growing, high-throughput bioanalytical platforms. Techniques for analyzing the metabolome are increasingly being applied to search for biomarkers and to connect genes and proteins with biological phenotypes. These techniques have also been useful for diagnosing diseases (Patel and Ahmed 2015; Rubert et al. 2017; Vincent et al. 2012), characterizing interactions between the pathogen and host (Kopka 2006; Tenenboim and Brotman 2016), and elucidating gene functions (Peyraud et al. 2017). The perception of external stimuli may induce changes to metabolites, usually before symptoms are observable. Thus, detecting these changes may be relevant for disease diagnoses during the latency stage of infection. Biomarkers have been applied to diagnose diseases in humans, including cancer, diabetes, and other metabolic diseases (Emwas et al. 2015; Maniscalco et al. 2018; Ussher et al. 2016). For plant diseases, there are many potential biomarkers related to host-pathogen systems, including plants affected by citrus yellow shoot disease (Cevallos-Cevallos et al. 2011; Killiny and Nehela 2017), grapevine infected with Botrytis cinerea (AbdelFarid et al. 2009; Hong et al. 2011; Xu et al. 2017), and plants infected with Fusarium oxysporum (Bollina et al. 2010; Prithiviraj et al. 2004). However, there have been relatively few reports describing the application of metabolomics for diagnosing plant diseases.

In this study, we employed a nontargeted metabolomics approach to profile the strawberry metabolome for an early diagnosis of strawberry anthracnose. We also aimed to characterize the biochemical mechanism underlying strawberry disease resistance during interactions with Colletotrichum theobromicola.

\section{Materials and Methods}

Chemicals and reagents. Analytical grade $\mathrm{N}, \mathrm{O}$-bis (trimethylsilyl) acetamide (containing 1\% TMS), pyridine, methoxyamine hydrochloride, and salicin (used as an internal standard) were purchased from Sigma-Aldrich (St. Louis, MO), while methyl alcohol was obtained from Sinopharm Chemical Reagent Co. Ltd 
(Beijing, China). Ultrapure water was prepared using the Milli-Q Academic A10 water purification system from Millipore (Billerica, MA).

Plant material and fungal strain. C. theobromicola strain 12 was isolated from plants grown in the Xiachang strawberry cultivation greenhouse in Fangshan, Beijing, China. Its identity was confirmed by PCR amplification and sequencing (Diao et al. 2015). Strawberry plants 'Hongyan' were purchased from the Xingshou Strawberry Cooperative (Beijing, China) and grown for 6 months in a greenhouse at China Agricultural University. C. theobromicola was grown on PDA medium in petri dishes incubated at $25^{\circ} \mathrm{C}$ until the mycelia covered the entire medium surface. A scalpel was then used to scratch the mycelia to promote sporulation. Conidia heap were transferred to a $50-\mathrm{ml}$ centrifuge tube containing $15 \mathrm{ml} 0.1 \%$ glucose and $0.05 \% \mathrm{vol} / \mathrm{vol}$ Tween-20 solution. The spore suspension was diluted to a concentration of $1 \times 10^{5}$ conidia/ml. Because the spores required darkness to germinate (Wastie 1972), strawberry plants grown in pots were inoculated in the evening. Specifically, 18 plants were sprayed with $25 \mathrm{ml}$ C. theobromicola spore suspension, and the control plants were sprayed with the solution containing same components but no spores. The pots were placed in a waterfilled tray and sealed with plastic film to maintain moist conditions. Strawberry plants were incubated at $25^{\circ} \mathrm{C}$ in darkness for $8 \mathrm{~h}$ and then placed under natural light in a greenhouse. The plastic film was removed after 3 days. Leaf samples were collected 3, 7, and 14 days after inoculation, with 6 replicates per treatment. For each replicate, leaves were cut from 6 plants using scissors sterilized with alcohol. The leaves were rinsed with distilled water to removal residual spores, dried, and then placed in liquid nitrogen to arrest enzymatic activity. The frozen leaf material was stored at $-80^{\circ} \mathrm{C}$. These were repeated twice.

Metabolome extraction. Metabolites were extracted from leaves according to an optimized published method (Heuberger et al. 2014a). Salicin (internal standard) was added to methanol/water (vol/vol, 4/1) to prepare a $10 \mu \mathrm{g} / \mathrm{ml}$ extraction solution. Frozen leaf samples were shaken in a ball mill to produce a homogenized powder, after which $100 \mathrm{mg}$ powder was added to a $2-\mathrm{ml}$ microcentrifuge tube. The powder was then suspended in $1.8 \mathrm{ml}$ extraction solution and the tube was mixed in the MX-F vortex mixer (Dragon Laboratory Instruments Co. Ltd., Beijing, China) for $1 \mathrm{~min}$ at room temperature. Samples were placed in an ultrasonic cleaner (Kunshan Ultrasonic Instruments Co. Ltd., Kunshan, China) for $20 \mathrm{~min}$ and then centrifuged (Eppendorf, Hauppauge, NY) at 15,000 $\times g$ for $15 \mathrm{~min}$. The supernatant, which contained the metabolites, was transferred to a new 0.6-ml microcentrifuge tube. The metabolome extraction step was repeated 6 times.

Metabolomic analysis. For the gas chromatography-mass spectrometry (GC-MS) analysis, $0.6 \mathrm{ml}$ extracted metabolite solution was dried using the ZLS-1 vacuum concentrator (Hunna Herexi Instrument $\&$ Equipment Co. Ltd., Hunan, China) and dissolved in $100 \mu$ l pyridine solution containing $20 \mathrm{mg} / \mathrm{ml}$ methoxyamine hydrochloride. Samples were incubated at $30^{\circ} \mathrm{C}$ for $2 \mathrm{~h}$, after which $100 \mu \mathrm{N}, O$-bis (trimethylsilyl) acetamide (containing $1 \%$ TMS) was added followed by an incubation at $37^{\circ} \mathrm{C}$ for $6 \mathrm{~h}$. Samples were centrifuged at $15,000 \times g$ for $15 \mathrm{~min}$ and then cooled to room temperature. Next, $160 \mu \mathrm{l}$ supernatant was transferred to a $250-\mu l$ glass insert in a GC-MS autosampler vial.

Metabolites were analyzed using the 7890-5975C GC-MS system (Agilent) equipped with a DB-5 capillary column $(30 \mathrm{~m} \times 250 \mu \mathrm{m} \times$ $0.25 \mu \mathrm{m})$. Samples $(1 \mu \mathrm{l})$ were injected by the Agilent autoinjector. Helium was used as the carrier gas, with a constant flow rate of $1 \mathrm{ml} / \mathrm{min}$. The GC oven was heated to $65^{\circ} \mathrm{C}$ for $2 \mathrm{~min}$, raised to $185^{\circ} \mathrm{C}$ at $5^{\circ} \mathrm{C} / \mathrm{min}$, raised to $200^{\circ} \mathrm{C}$ at $1^{\circ} \mathrm{C} / \mathrm{min}$, raised to $280^{\circ} \mathrm{C}$ at $15^{\circ} \mathrm{C} / \mathrm{min}$, and then maintained at $280^{\circ} \mathrm{C}$ for $5 \mathrm{~min}$. The ion source and ion source surface temperatures were set to $230^{\circ} \mathrm{C}$ and $280^{\circ} \mathrm{C}$, respectively. Electron impact ionization $(70 \mathrm{eV})$ was set in the full scan mode $(\mathrm{m} / \mathrm{z}, 20$ to 650$)$ at $0.2 \mathrm{~s} / \mathrm{scan}$. The acceleration voltage was turned on after a solvent delay of $4 \mathrm{~min}$. Qualitative Analysis B.07.00 (Agilent) was used to acquire mass spectrometric data. The mass spectra of all detected compounds were compared with spectra in the NIST14 library, and metabolites were tentatively identified based on one quantitative ion and two reference ions. Relative metabolite contents were determined according to the quantitative ion peak area.

Data analysis. We used SPSS Statistics 21.0 (IBM) and Unscrambler10.3 (CAMO, Trondheim, Norway) for a multivariate statistical analysis. Data for specific metabolites underwent a one-way analysis of variance (ANOVA) to assess the significance of metabolic changes; the differences were considered significant at $P<0.05$. The data also underwent a principal component analysis (PCA) and partial least squares discriminant analysis (PLS-DA). Sample quality was assessed by examining the values in the PCA plot. The PCA calculated all of the possible principal components, which were then visually presented in a score plot. The scores were used to assess data quality (López-Gresa et al. 2010). Sample outliers were detected in the PCA score plots. The partial least squares (PLS) is a regression method commonly used in multivariate studies in order to find the relationship between two data tables. The orthogonal partial least squares (OPLS) is an optimization of PLS, which separates the systematic variation in $X$ into two parts, one that is linearly related to $Y$ and one that is orthogonal to $Y$. To identify the metabolites that were associated with infection, a supervised PLS-DA was subsequently completed.

The PLS-DA was applied to diagnose strawberry anthracnose according to a published method (Cynkar et al. 2010; Lvova et al. 2012; Manach et al. 2004). The model used one response variable $(Y)$, which codes for class membership as follows: 1 for members of the diseased plants, 0 for members of the healthy plants. Twothirds of the test samples known to belong to two tribes (diseased plants and healthy plants) were used to build a discriminant model, while one-third of the test samples of known origin made up a test set to be classified. The PLS-DA was useful for cases with more than two classes. Each class was represented by a categorical variable (i.e., a binary variable with a value of 1 for members of that class, and a value of 0 for nonmembers). The calculation procedure was followed as described (Kalivodová et al. 2015):

$$
Y=X B+E
$$

$X$ was the sample spectral matrix, $Y$ was the response output matrix, and $B$ and $E$ represent the regression coefficients matrix and error matrix, respectively.

By building a PLS-DA model with all categorical variables as $Y$, we were able to directly predict class memberships from the $X$-variables describing the samples.

Ypred $>0.5$ means "approximating 1" (i.e., member);

Ypred $<0.5$ means "approximating 0" (i.e., nonmember).

After the PLS model was checked and validated, we predicted the classifications of new samples. The prediction results were interpreted by viewing the Predicted with Deviations plot for each class indicator $Y$-variable:

Samples with Ypred $>0.5$ and a deviation that did not exceed 0.5 were predicted to be members;

Samples with Ypred $<0.5$ and a deviation that did not exceed 0.5 were predicted to be nonmembers;

Samples with a deviation that exceeded 0.5 were not classified.

\section{Results}

Analysis of anthracnose-infected strawberry plants. Leaves were collected from strawberry plants inoculated with $C$. theobromicola or control solution at 3, 7, and 14 days after the inoculation (Fig. 1 ). The fungus-induced speckle symptoms were only observed on strawberry leaves after 7 or 14 days of inoculation. In the absence of visible symptoms, diseased plants (3-day-inoculated strawberry plants) were difficult to distinguish from healthy plants. The 7-dayinoculated leaves showed obvious spots and grew weaker. Another 7 days later, spots covered almost the entire leaf surface and leaves and stems were visibly wilting.

Samples corresponding to typical GC-MS total ion chromatogram (TIC) peaks were collected at different time points (Fig. 2) 
Metabolites were extracted from strawberry leaves and detected by GC-MS. We detected 189 and 202 TIC peaks for control and inoculated plants, respectively. The majority of the peaks in the chromatograms were identified as endogenous metabolites by NIST mass spectra library, including organic acids, amino acids, carbohydrates, alcohols, polyphenols, and other metabolites.

Principal component analysis and orthogonal partial least squares analysis based on the metabolic fingerprints of control and inoculated plants. Metabolomics data for samples at different time points was subject to PCA (Fig. 3). In this analysis, the plot labels showed that PC-1 explained $43 \%$ and PC-2 $12 \%$ of the total variance in the data. Compared with the control group that had a value distribution from -7.9 to -3.3 on PC1, the inoculated group, especially the ones sampled on the 7th and 14th day following inoculation ranged from -0.5 to 17.5 on PC1. During the examination of all samples in a workset, the PCA score plot represented the sample distribution in the new multivariate space, in which different groups were clearly separated.

OPLS analysis supervised learning method was used to analyze GC-MS data. During examination of samples collected at three time points from inoculated and control plants, an OPLS score plot (Fig. 4) represented the sample distribution in the new multivariate space, with different groups clearly separated. The sum of the explained $X$ variances $(52 \%)$ explained considerable portion of the variance in $Y(95 \%)$. There was distinct discrimination between inoculated and healthy plants as time after inoculation increased along the Factor- 1 axis. These results implied that the inoculation with $C$. theobromicola induced metabolic changes even before disease symptoms appeared. Moreover, the metabolic changes were associated with the degree to which the pathogen spread throughout plants.

The correlation loading plot of the predictive component of the OPLS based on the metabolite profiles of strawberry leaves 3, 7, and 14 days after inoculation with $C$. theobromicola or treated with the control solution showed that some variables were closest to the response variable class between the two circles (Fig. 5). The correlation loading plot contained two ellipses that indicated how much variance was considered by the model. The outer ellipse represented the unit-circle, corresponding to $100 \%$ of the explained variance, while the inner ellipse corresponded to $50 \%$ of the explained variance. The metabolites (e.g., glucitol and shikimic acid) close to the outer ellipse were important for distinguishing between the inoculated and control samples.

Identification of diseased and healthy plants with the partial least squares discrimination analysis model. PLS-DA score plot of the metabolome of strawberry leaves inoculated with C. theobromicola or treated with the control solution was shown in Figure 6. Results showed that the two classes were perfectly separated with $48 \%$ of the $X$ variance explaining $94 \%$ of the $Y$ variance from Factor-1 and Factor-2. The PLS-DA is based on the PLS method, and is used to establish a regression model for sample category variables and variables. Of the 35 collected samples, 24 were selected as the training set and 11 were selected as the test set according to a 2:1 ratio for the training and test sets. The subsequently established PLSDA model enabled the clear separation of the anthracnose-infected strawberry plants (diseased plants) from the healthy plants. Additionally, with $48 \%$ of the $X$ variance explaining $94 \%$ of the $Y$ variance, the model was considered reliable and useful for a discriminant analysis.

The relationship between predicted and reference category variables of diseased and healthy samples based on the PLS-DA model was shown in Figure 7. The root mean squared errors (RMSEs) were 0.0335 and 0.1446 (close to 0 ), while the offsets were 0.0022 and 0.0873 (close to 0) and the slopes were 0.9955 and 0.8193 (close to 1). The two straight lines represented the regression analysis of the model calibration data and the validation results. The two regression lines basically coincided. The plot presents points close to a straight line through the origin and a slope close to 1 . These RMSEs tending to 0 made the PLS-DA model fit the data.

The model was used for the predictions of 11 samples (Fig. 8). PLS-DA of healthy and diseased strawberry plants showed that the values of predicted $Y$ in test set were 0.68 to 1.51 for the group
$3 d$
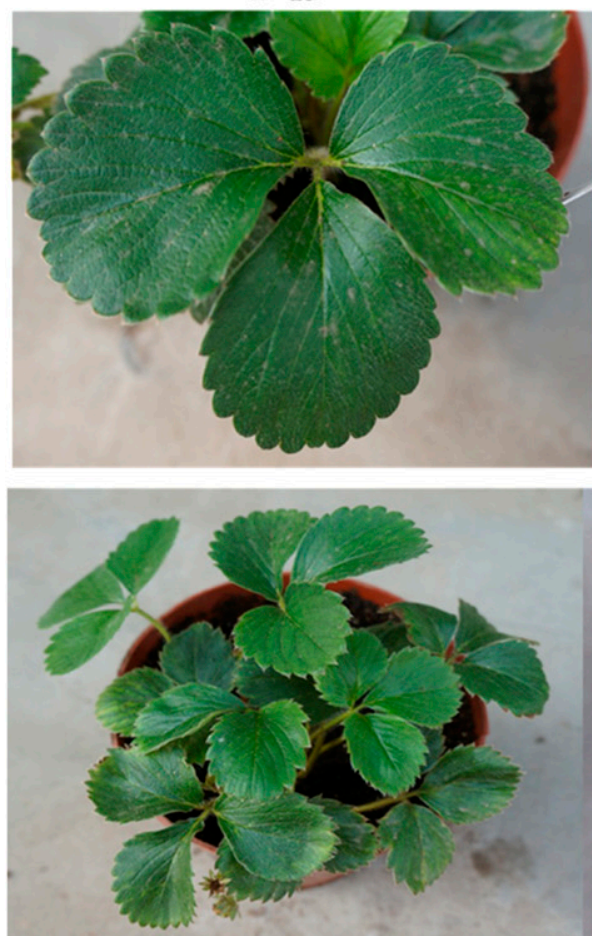

$7 d$

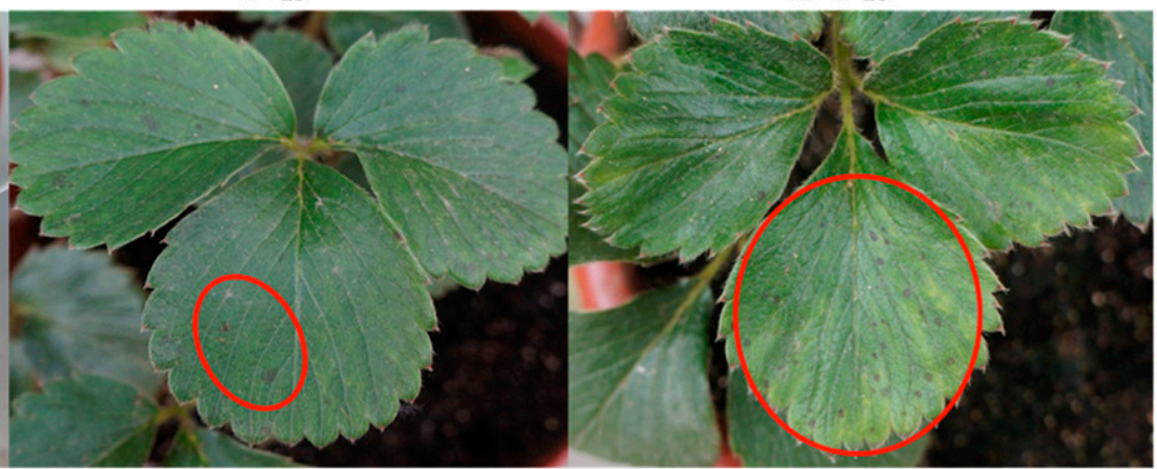

Fig. 1. Strawberry plants of 'Hongyan' cultivar were inoculated with Colletotrichum theobromicola and sampled after 3, 7, and 14 days. The brown spots appeared on the leaves 7 days after inoculation, and the arrows refer to the blight of the stems and leaves. Strawberry plants were inoculated with a $1 \times 10^{5}$ conidia/ml spore suspension. Six replicates of plant samples were detected per treatment. 
inoculated with $C$. theobromicola, and 0.11 to 0.27 for the healthy group. All of the samples exhibited a low prediction bias (close to 0.1 ). Additionally, the Ypred of samples 1 to 6 was greater than 0.5 (close to 1), and these samples were classified as diseased samples. Meanwhile, the Ypred of samples 7 to 11 was less than 0.5 (close to 0), implying these samples were the healthy samples. All 11 samples were correctly discriminated (i.e., 100\% accuracy).

Analysis of the untargeted metabolome and biomarkers interpretation. In the correlation loading plot, the variables spreading on the Factor-1 axis within 50\% 100\% variance ellipse are hopefully to contribute for the separation of the inoculated and control plant samples. These variables are important for modeling the response, and may be the biomarkers metabolites for strawberry interaction with C. theobromicola. According to the OPLS analysis and ANOVA, 36, 97 , and 106 significant peaks were detected for the leaf samples collected 3,7 , and, 14 days after inoculation, respectively. On the basis of a qualitative analysis involving standard compounds and the NIST library (match $>80 \%$ ), the first three fragment-ion $\mathrm{m} / \mathrm{z}$ values with the highest abundance within each fragmentation pattern and 20 metabolites were identified as biomarkers (Table 1). In the 3-day sample, 8 metabolites

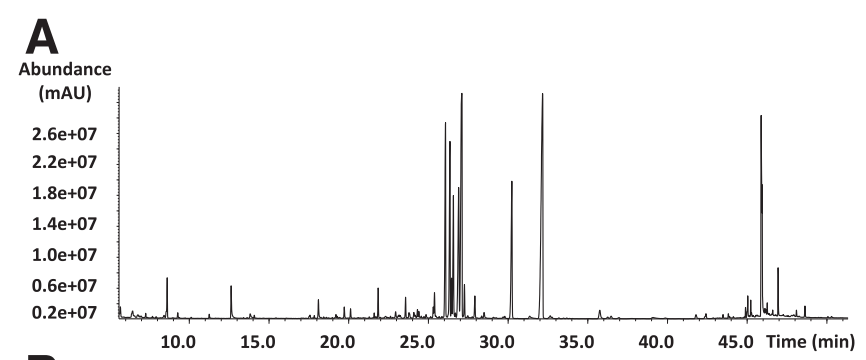

B

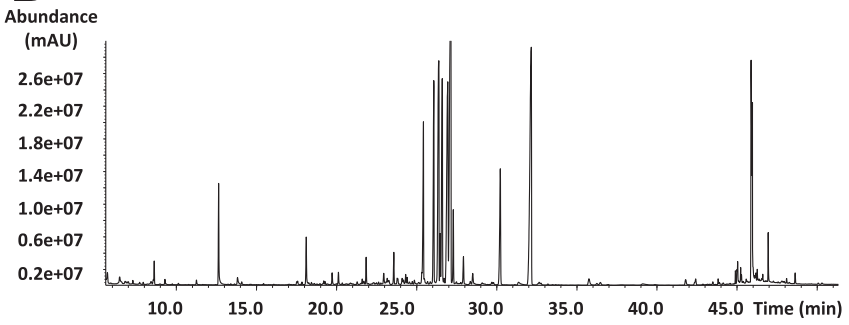

Fig. 2. Representative gas chromatography-mass spectrometry total ion chromatograms of metabolome extracted from the strawberry plants 'Hongyan' inoculated with (A) or without (B) Colletotrichum theobromicola spore suspension. The spore suspension was diluted to be $10^{5}$ conidia/ml in $0.1 \%$ glucose and $0.05 \%$ Tween-20 aqueous solution and sprayed on the leaves. The metabolome of strawberry leaves were extracted with a mixture of methanol/water $(4: 1, \mathrm{vol} / \mathrm{vol})$, with six replicates per treatment. were decreased, while 12 were elevated. In the 7- and 14-day samples, 9 metabolites were decreased and 11 were elevated.

\section{Discussion}

The OPLS analysis offered a good separation for control plants and inoculated ones, and the relationships were interpreted with a much higher degree of certainty compared with PCA.

Furthermore, there was less variability in the scores for the healthy plants than in the scores for the diseased plants, suggesting there were differences among the leaf samples collected from the diseased plants at three time points. The PLS-DA involves the application of PLS regression for discriminating or classifying samples. Some available statistics may be used to assess the quality of the regression. All samples were correctly discriminated, indicating the PLS-DA model may be useful for differentiating between diseased and healthy strawberry plants.

In this study, some metabolites in Table 1 (e.g., gallic acid, phosphate, malic acid, citric acid, and erythrose) were elevated prior to the appearance of anthracnose symptoms on strawberry leaves but were decreased after symptoms appeared. These observations may be related to the early defense response of plants. Gallic acid is a polyphenol commonly found in plants and is considered to be a secondary metabolite (Manach et al. 2004). Earlier investigations revealed that such metabolites have antibacterial and antidisease effects (Soobrattee et al. 2005). Moreover, natural phenols may exhibit antibiofouling properties (Sendamangalam et al. 2011). After a pathogen infects

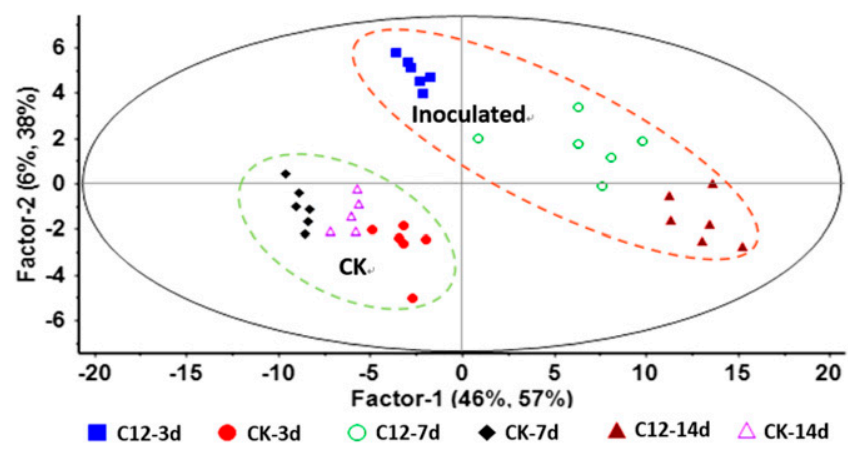

Fig. 4. Orthogonal partial least squares score plot for the leaves collected 3,7 , and 14 days after being inoculated with Colletotrichum theobromicola spore suspension $(\mathbf{\square}, \bigcirc, \boldsymbol{\Delta})$ or treated with blank solution $(\bullet,-\Delta)$. The scores plot illustrating $95 \%$ confidence ellipse for data having one predictive. The ellipse defines the statistical significance of class separation and provides an illustration where two groups represent control and inoculated plants.

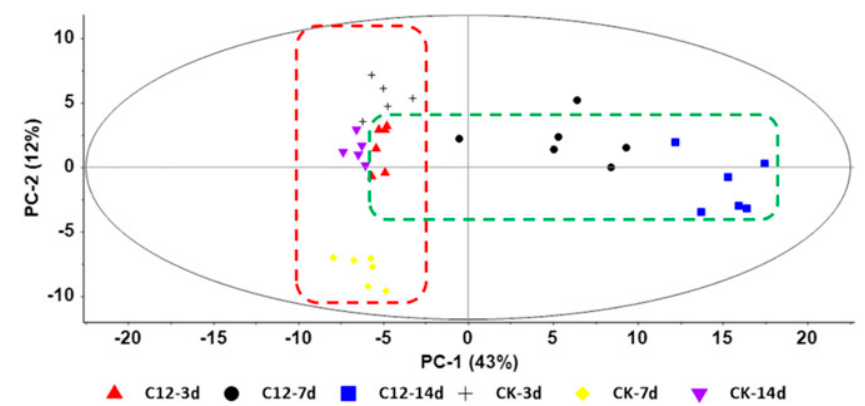

Fig. 3. Principal component analysis score plot of the metabolite profiles of strawberry leaves inoculated with Colletotrichum theobromicola spore suspension $(\mathbf{\Lambda}, \bullet, \mathbf{\bullet})$ or blank solution $(+,-, \mathbf{v})$ after 3,7 , and 14 days respectively. The control group (red dotted box) and the inoculated group (green dotted box) have a potential to separate on PCA score plot.

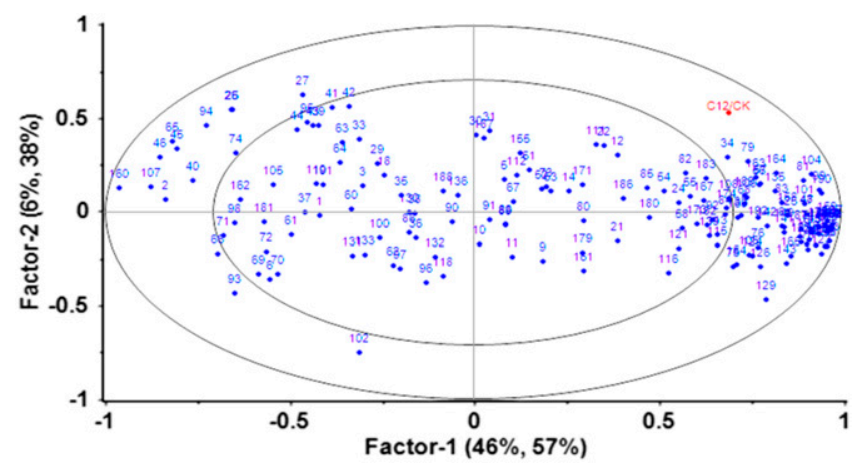

Fig. 5. Correlation loading plot of the predictive component of the orthogonal partial least squares based on the metabolite profiles of strawberry leaves 3,7 , and 14 days after inoculation with Colletotrichum theobromicola spore suspension and blank solution. The variables closest to the response variable (red point, C12/CK) and between the $50 \%$ and $100 \%$ explained circles are the most important variables that contribute to separation of the control and inoculated plants. 
a plant, the production of ROS prevents the pathogen from spreading (Egan et al. 2007). Defense-related signals induce the production of NADP-malic enzyme, which catabolizes malic acid to produce $\mathrm{NADPH}$, which can be used to produce $\mathrm{H}_{2} \mathrm{O}_{2}$ (Casati et al. 1999; Parker et al. 2009). Malic acid and citric acid also mediate stomatal closure and leaf rolling, with implications for plant defenses (Heuberger et al. 2014b; Saglam et al. 2010).

Galacturonic acid is the main structural component of pectic polymers, which are important for the production of the middle lamella and primary cell wall of higher plants (Prade et al. 1999). Pectin can prevent pathogens from invading plants, while also serving as a carbon source for plants. Additionally, the products of pectin degradation can induce defense responses in plants (González and Allen 2003; Ridley et al. 2001). In our study, galacturonic acid concentrations were more than two-fold higher in diseased plants than in healthy plants. This suggests that $C$. theobromicola induces plant defense responses as well as structural changes in the middle lamella and primary cell wall of plants.

Shikimic acid is an important precursor of phenylpropanoids, which can strengthen cell walls to enhance disease resistance during the early stages of pathogen infection (Huckelhoven 2007; Parker et al. 2009). However, our data revealed a significant decrease in shikimic acid, galactose, and D-xylose contents in inoculated strawberry

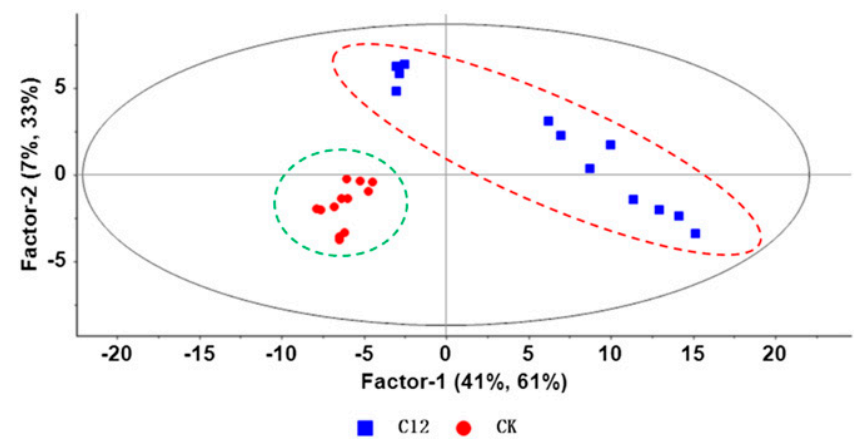

Fig. 6. Partial least squares discrimination analysis score plot of the metabolome of strawberry leaves inoculated with Colletotrichum theobromicola spore suspension (C12 a) and with blank solution (CK $\bullet$ ). Factor-1 and Factor-2 refer to the first two factors used to explain the variance. The scores plot illustrating $95 \%$ confidence ellipse for data having one predictive. The ellipse defines the statistical significance of class separation and provides an illustration where two groups represent healthy and diseased plants.

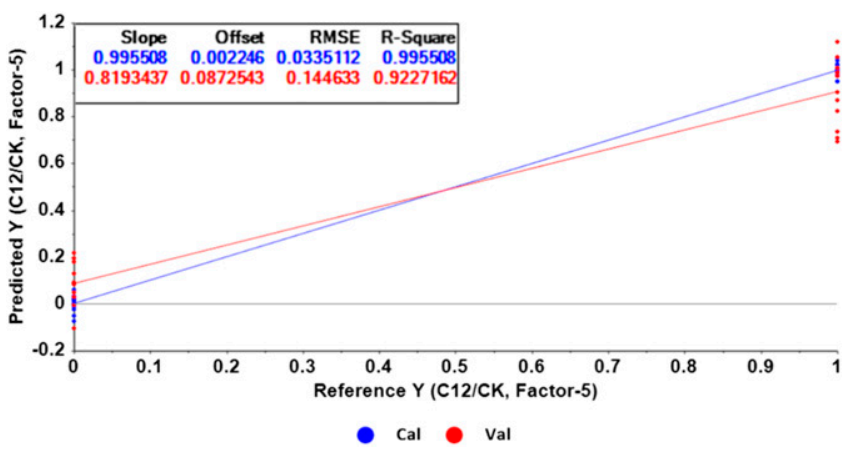

Fig. 7. Relationship between predicted and reference category variables of anthracnose-infected strawberry and control samples based on the Partial least squares discriminant analysis model. C12, treatments; CK, controls; Cal, calibration; Val, validation; $\mathrm{C} 12 / \mathrm{CK}$, response variables $\mathrm{Y}$; Factor-5, five factors used to explain the variance. The blue points are from calibration involving re-sampling with replacement. The red points are from cross validation which is more conservative as the sample was not a part of the model when it was predicted. The two regression lines basically coincided and closed to a straight line through the origin with a slope close to 1 indicated it to be a reliable model. leaves. These changes may be related to the colonization of strawberry plants by $C$. theobromicola.

We observed an increase in sucrose content in the inoculated strawberry leaves. Results of an analogous study concerning barley powdery mildew indicated that invertase activity increases in response to infections, resulting in the accumulation of hexose and sucrose as well as decreased photosynthetic activities (Swarbrick et al. 2006). These changes may be due to the spread of pathogens and associated increase in damage. Pathogens can affect the energy usage and production of carbon sources (Thines et al. 2000) (e.g., sucrose, glucose, and fructose [Roitsch and González 2004]) in plant hosts. Sucrose is produced during photosynthesis and is involved in the synthesis of storage reserves in higher plants (Tauzin et al. 2014). As a signaling molecule, sucrose affects plant development (Tognetti et al. 2013), and the observed increase in sucrose content indicates that the normal growth of strawberry plants may be disturbed during an infection by $C$. theobromicola.

Inorganic phosphates function as structural components, while also regulating enzyme activity and gene expression. Thus, they are important for plant growth. Additionally, inorganic phosphates maintain homeostasis in plants and can be stored and transported within cells (Mimura 1995). Our data indicated that C. theobromicola can increase inorganic phosphate levels by more than twofold compared with midlate stages during the early stages of infection, which is followed by a decrease in inorganic phosphate content.

We established a reliable and highly accurate PLS-DA model that can discriminate between control plants and plants infected by $C$. theobromicola. This model may be useful for diagnosing anthracnose in strawberry plants during the early stages of infection. Strawberry plantations often have a risk of potential disease infection, and an early diagnosis of strawberry anthracnose may have a series of benefits on plant protection. Firstly, it may help farmers to discover the disease in its latent period, which will reduce the occurrence of largescale plant disease and yield loss. Early diagnosis may also offer guidance on the selection of fungicide modes of action most appropriate for disease control and avoid an excessive prophylactic application that may cause resistance or environmental pollution. Finally, disease diagnosis before transplanting is of great importance to prevent spread of anthracnose from the nurseries to the plantation farms.

Additionally, we observed considerable changes in the metabolite contents of strawberry plants that were induced by $C$. theobromicola. The affected metabolites represent tentative biomarkers for the early stages of strawberry anthracnose. Further analyses indicated these biomarkers are closely related to the spread of pathogens in plants as well as plant disease resistance. Moreover, the altered regulation

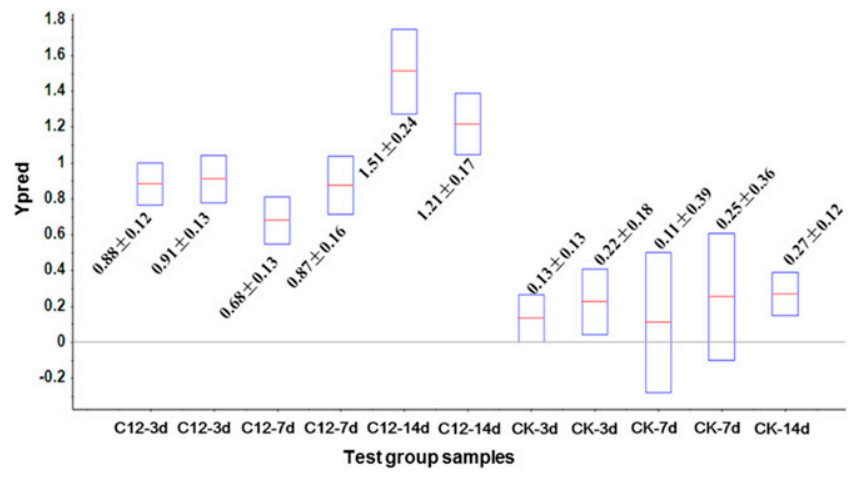

Fig. 8. Partial least squares discriminant analysis between healthy and diseased strawberry plants inoculated after 3, 7, and 14 days, respectively. Samples with Ypred $>0.5$ and a deviation of $\leq 0.5$ were predicted to be diseased plants; samples with Ypred $<0.5$ and a deviation of $\leq 0.5$ were predicted to be healthy plants; samples with a deviation that exceeded 0.5 were not classified. The values of predicted $Y$ in test set were 0.68 to 1.51 for the group inoculated with Colletotrichum theobromicola, and 0.11 to 0.27 for the control group showed a $100 \%$ correct diagnosis of diseases by this method. 
Table 1. Potential biomarkers ${ }^{\mathrm{a}}$ for infection of Colletotrichum theobromicola in strawberry plants

\begin{tabular}{|c|c|c|c|c|c|c|c|}
\hline \multirow[b]{2}{*}{ Metabolites } & \multirow{2}{*}{$\begin{array}{c}\mathbf{R T}^{\mathbf{b}} \\
(\mathbf{m i n})\end{array}$} & \multicolumn{3}{|c|}{$\mathbf{m} / \mathbf{z}$} & \multicolumn{3}{|c|}{ Fold change $^{c}$} \\
\hline & & No. 1 & No. 2 & No. 3 & 3 days & 7 days & 14 days \\
\hline Phosphorate & 12.63 & 299 & 314 & 133 & $2.08 *$ & $0.55^{*}$ & 0.77 \\
\hline Glyceric acid & 14.04 & 147 & 189 & 292 & 1.03 & 1.05 & $1.29 *$ \\
\hline Malic acid & 18.10 & 233 & 147 & 245 & $2.31 *$ & $0.59 *$ & 0.74 \\
\hline Erythrose & 18.43 & 205 & 147 & 117 & $8.72 *$ & $0.55^{*}$ & 0.80 \\
\hline Tartaric acid & 21.53 & 147 & 292 & 219 & 0.83 & $0.64 *$ & $0.78^{*}$ \\
\hline D-Xylose & 21.85 & 103 & 307 & 217 & $0.70 *$ & $0.66^{*}$ & 0.79 \\
\hline D-Ribose & 23.78 & 217 & 204 & 191 & 1.07 & $1.61 *$ & $1.68 *$ \\
\hline Shikimic acid & 25.31 & 204 & 147 & 255 & $0.70 *$ & $0.67 *$ & 0.85 \\
\hline Citric acid & 25.39 & 273 & 147 & 363 & $4.02 *$ & $0.20 *$ & 0.36 \\
\hline Galactose & 26.70 & 319 & 205 & 147 & $0.03 *$ & 0.56 & 0.86 \\
\hline Methyl $\alpha$-D-galactopyranoside & 27.10 & 204 & 217 & 133 & $1.13 *$ & $1.26^{*}$ & $1.31 *$ \\
\hline Glucitol & 27.53 & 319 & 205 & 147 & 0.70 & 1.38 & $2.69 *$ \\
\hline Gallic acid & 28.50 & 281 & 458 & 443 & $1.38 *$ & 0.82 & 0.97 \\
\hline Phytol & 35.76 & 143 & 75 & 144 & 0.81 & 1.21 & $1.61 *$ \\
\hline D-Mannopyranose & 36.25 & 204 & 191 & 75 & 1.10 & $2.20 *$ & $3.31 *$ \\
\hline$\beta$-D-Glucopyranose & 43.49 & 204 & 191 & 147 & 1.23 & $1.99 *$ & $2.71 *$ \\
\hline Sucrose & 45.04 & 361 & 362 & 217 & 1.16 & $1.21 *$ & $1.42 *$ \\
\hline Rhodioloside & 46.94 & 204 & 193 & 217 & 1.02 & $2.24 *$ & $6.16^{*}$ \\
\hline Galacturonic acid & 48.63 & 204 & 217 & 147 & 0.84 & $3.30 *$ & $2.10 *$ \\
\hline Melibiose & 50.30 & 204 & 217 & 191 & 0.89 & $3.52 *$ & 1.49 \\
\hline
\end{tabular}

a The metabolites contents changed significantly $(P \leq 0.05)$ in inoculated plants at least once out of three sampling times compared with those in noninoculated ones by one-way ANOVA.

b Abbreviations: $\mathrm{RT}=$ Retention time, $\mathrm{m} / \mathrm{z}=$ Mass-to-charge ratio.

c The fold of metabolites contents changed in $C$. theobromicola inoculated plants compared with noninoculated ones after the same days of inoculation. The numbers greater and less than 1 represent the elevation and decrease of metabolites, respectively. "*” represents a significant difference $(P \leq 0.05)$ existing in the metabolite content between inoculated and control plants by one-way ANOVA.

of metabolic pathways, including galactose, starch, sugar, and amino acid metabolism, leads to phenotypic changes influencing disease resistance or the development of necrosis. Thus, a combination of PLS-DA, OPLS, and ANOVA may be necessary for analyzing metabolomics data. The results of these analyses may provide an important reference for disease diagnosis and plant-pathogen interactions.

\section{Acknowledgments}

The authors thank Qiliang Huang and Lidong Cao of Key Laboratory of Integrated Pest Management in Crops, Ministry of Agriculture, Institute of Plant Protection, Chinese Academy of Agricultural Sciences for providing the instruments used for metabolite detection. The authors also thank Guozhen Zhang of the Key Laboratory for Biological Monitoring and Green Control of China Agricultural University for guidance on methods of biological test.

\section{Literature Cited}

Abdel-Farid, I. B., Jahangir, M., van den Hondel, C. A. M. J. J., Kim, H. K., Choi, Y. H., and Verpoorte, R. 2009. Fungal infection-induced metabolites in Brassica rapa. Plant Sci. 176:608-615.

Bollina, V., Kumaraswamy, G. K., Kushalappa, A. C., Choo, T. M., Dion, Y., Rioux, S., Faubert, D., and Hamzehzarghani, H. 2010. Mass spectrometry-based metabolomics application to identify quantitative resistance-related metabolites in barley against Fusarium head blight. Mol. Plant Pathol. 11:769-782.

Brooks, A. N. 1931. Anthracnose of strawberry caused by Colletotrichum fragariae, n. sp. Phytopathology 21:739-744.

Buddie, A. G., Martinez-Culebras, P., and Bridge, P. D. 1999. Molecular characterization of Colletotrichum strains derived from strawberry. Mycol. Res. 103:385-394.

Casati, P., Drincovich, M. F., Edwards, G. E., and Andreo, C. S. 1999. Malate metabolism by NADP-malic enzyme in plant defense. Photosynth. Res. 61: 99-105.

Cevallos-Cevallos, J. M., García-Torres, R., Etxeberria, E., and Reyes-DeCorcuera, J. I. 2011. GC-MS analysis of headspace and liquid extracts for metabolomic differentiation of Citrus Huanglongbing and zinc deficiency in leaves of 'Valencia' sweet orange from commercial groves. Phytochem. Anal. 22:236-246.

Cynkar, W., Dambergs, R., Smith, P., and Cozzolino, D. 2010. Classification of Tempranillo wines according to geographic origin: Combination of mass spectrometry based electronic nose and chemometrics. Anal. Chim. Acta 660: 227-231.

Diao, Y., Zhang, C., Xu, J., Lin, D., Liu, L., Mtung'e, O. G., and Liu, X. 2015. Genetic differentiation and recombination among geographic populations of the fungal pathogen Colletotrichum truncatum from chili peppers in China. Evol. Appl. 8:108-118.

Egan, M. J., Wang, Z. Y., Jones, M. A., Smirnoff, N., and Talbot, N. J. 2007. Generation of reactive oxygen species by fungal NADPH oxidases is required for rice blast disease. P. Natl. Acad. Sci. 104:11772-11777.

Emwas, A.-H., Luchinat, C., Turano, P., Tenori, L., Roy, R., Salek, R. M., Ryan, D., Merzaban, J. S., Kaddurah-Daouk, R., Zeri, A. C., Gowda, G. A. N., Raftery, D., Wang, Y., Brennan, L., and Wishart, D. S. 2015. Standardizing the experimental conditions for using urine in NMR-based metabolomic studies with a particular focus on diagnostic studies: A review. Metabolomics 11:872-894.

Fiehn, O., Kopka, J., Dörmann, P., Altmann, T., Trethewey, R. N., and Willmitzer, L. 2000. Metabolite profiling for plant functional genomics. Nat. Biotechnol. 18:1157-1161.

Freeman, S., Horowitz, S., and Sharon, A. 2001. Pathogenic and nonpathogenic lifestyles in Colletotrichum acutatum from strawberry and other plants. Phytopathology 91:986-992.

Gareau, B. J. 2008. Dangerous holes in global environmental governance: The roles of neoliberal discourse, science, and California agriculture in the Montreal Protocol. Antipode 40:102-130.

González, E. T., and Allen, C. 2003. Characterization of a Ralstonia solanacearum operon required for polygalacturonate degradation and uptake of galacturonic acid. Mol. Plant-Microbe Interact. 16:536-544.

Heuberger, A. L., Broeckling, C. D., Kirkpatrick, K. R., and Prenni, J. E. 2014a. Application of nontargeted metabolite profiling to discover novel markers of quality traits in an advanced population of malting barley. Plant Biotechnol. J. 12:147-160.

Heuberger, A. L., Robison, F. M., Lyons, S. M. A., Broeckling, C. D., and Prenni, J. E. 2014b. Evaluating plant immunity using mass spectrometry-based metabolomics workflows. Front. Plant Sci. 5:291.

Hong, Y. S., Cilindre, C., Liger-Belair, G., Jeandet, P., Hertkorn, N., and SchmittKopplin, P. 2011. Metabolic influence of Botrytis cinerea infection in champagne base wine. J. Agric. Food Chem. 59:7237-7245.

Huckelhoven, R. 2007. Cell wall-associated mechanisms of disease resistance and susceptibility. Annu. Rev. Phytopathol. 45:101-127.

Israel, D. W., Giddens, J. E., and Powell, W. W. 1973. The toxicity of peach tree roots. Plant Soil 39:103-112.

Kalivodová, A., Hron, K., Filzmoser, P., Najdekr, L., Janečková, H., and Adam, T. 2015. PLS-DA for compositional data with application to metabolomics. J. Chemometr. 29:21-28.

Killiny, N., and Nehela, Y. 2017. Metabolomic response to Huanglongbing: Role of carboxylic compounds in Citrus sinensis response to 'Candidatus Liberibacter asiaticus' and its vector, Diaphorina citri. Mol. Plant-Microbe Interact. 30:666-678

Kopka, J. 2006. Current challenges and developments in GC-MS based metabolite profiling technology. J. Biotechnol. 124:312-322. 
López-Gresa, M. P., Maltese, F., Bellés, J. M., Conejero, V., Kim, H. K., Choi, Y. H., and Verpoorte, R. 2010. Metabolic response of tomato leaves upon different plant-pathogen interactions. Phytochem. Anal. 21:89-94.

Lvova, L., Denis, S., Barra, A., Mielle, P., Salles, C., Vergoignan, C., Natale, C. D., Paolesse, R., Temple-Boyer, P., and Feron, G. 2012. Salt release monitoring with specific sensors in "in vitro" oral and digestive environments from soft cheeses. Talanta 97:171-180.

Manach, C., Scalbert, A., Morand, C., Rémésy, C., and Jiménez, L. 2004. Polyphenols: Food sources and bioavailability. Am. J. Clin. Nutr. 79:727-747.

Maniscalco, M., Paris, D., Melck, D. J., Molino, A., Carone, M., Ruggeri, P., Caramori, G., and Motta, A. 2018. Differential diagnosis between newly diagnosed asthma and COPD using exhaled breath condensate metabolomics: A pilot study. Eur. Respir. J. 51:1701825.

Mimura, T. 1995. Homeostasis and transport of inorganic phosphate in plants. Plant Cell Physiol. 36:1-7.

Parker, D., Beckmann, M., Zubair, H., Enot, D. P., Caracuel-Rios, Z., Overy, D. P., Snowdon, S., Talbot, N. J., and Draper, J. 2009. Metabolomic analysis reveals a common pattern of metabolic re-programming during invasion of three host plant species by Magnaporthe grisea. Plant J. 59:723-737.

Patel, S., and Ahmed, S. 2015. Emerging field of metabolomics: Big promise for cancer biomarker identification and drug discovery. J. Pharm. Biomed. Anal. 107:63-74.

Peyraud, R., Dubiella, U., Barbacci, A., Genin, S., Raffaele, S., and Roby, D. 2017. Advances on plant-pathogen interactions from molecular toward systems biology perspectives. Plant J. 90:720-737.

Prade, R. A., Zhan, D., Ayoubi, P., and Mort, A. J. 1999. Pectins, pectinases and plant-microbe interactions. Biotechnol. Genet. Eng. Rev. 16:361-392.

Prithiviraj, B., Vikram, A., Kushalappa, A. C., and Yaylayan, V. 2004. Volatile metabolite profiling for the discrimination of onion bulbs infected by Erwinia carotovora ssp. carotovora, Fusarium oxysporum and Botrytis allii. Eur. J. Plant Pathol. 110:371-377.

Ridley, B. L., O'Neill, M. A., and Mohnen, D. 2001. Pectins: Structure, biosynthesis, and oligogalacturonide-related signaling. Phytochemistry 57:929-967.

Roessner, U., Wagner, C., Kopka, J., Trethewey, R. N., and Willmitzer, L. 2000. Simultaneous analysis of metabolites in potato tuber by gas chromatography-mass spectrometry. Plant J. 23:131-142.

Roitsch, T., and González, M. C. 2004. Function and regulation of plant invertases: Sweet sensations. Trends Plant Sci. 9:606-613.

Rubert, J., Righetti, L., Stranska-Zachariasova, M., Dzuman, Z., Chrpova, J., Dall'Asta, C., and Hajslova, J. 2017. Untargeted metabolomics based on ultra-high-performance liquid chromatography-high-resolution mass spectrometry merged with chemometrics: A new predictable tool for an early detection of mycotoxins. Food Chem. 224:423-431.

Saglam, A., Terzi, R., Nar, H., Saruhan, N., Ayaz, F., and Kadioglu, A. 2010. Inorganic and organic solutes in apoplastic and symplastic spaces contribute to osmotic adjustment during leaf rolling in Ctenanthe setosa. Acta Biol. Cracov. Ser.; Bot. 52:37-44.

Sendamangalam, V., Choi, O. K., Kim, D., and Seo, Y. 2011. The antibiofouling effect of polyphenols against Streptococcus mutans. Biofouling 27:13-19.

Sewell, G. W. F. 1979. Reappraisal of the nature of the specific replant disease of apple. Rev. Plant Pathol. 58:209-211.

Simpson, D. 2018. The economic importance of strawberry crops. Pages 1-7 in: The Genomes of Rosaceous Berries and Their Wild Relatives. Springer, Cham.

Soobrattee, M. A., Neergheen, V. S., Luximon-Ramma, A., Aruoma, O. I., an Bahorun, T. 2005. Phenolics as potential antioxidant therapeutic agents: Mechanism and actions. Mutat. Res. 579:200-213.

Swarbrick, P., Schulze-Lefert, P., and Scholes, J. 2006. Metabolic consequences of susceptibility and resistance (race-specific and broad spectrum) in barley leaves challenged with powdery mildew. Plant Cell Environ. 29:1061-1076.

Tauzin, A. S., Sulzenbacher, G., Lafond, M., Desseaux, V., Reca, I. B., Perrier, J. Bellincampi, D., Fourquet, P., Lévêquee, C., and Giardina, T. 2014. Functional characterization of a vacuolar invertase from Solanum lycopersicum: Posttranslational regulation by $\mathrm{N}$-glycosylation and a proteinaceous inhibitor. Biochimie 101:39-49.

Tenenboim, H., and Brotman, Y. 2016. Omic relief for the biotically stressed: Metabolomics of plant biotic interactions. Trends Plant Sci. 21:781-791.

Thines, E., Weber, R. W., and Talbot, N. J. 2000. MAP kinase and protein kinase A-dependent mobilization of triacylglycerol and glycogen during appressorium turgor generation by Magnaporthe grisea. Plant Cell 12:1703-1718.

Tognetti, J. A., Pontis, H. G., and Martinez-Noel, G. M. 2013. Sucrose signaling in plants: A world yet to be explored. Plant Signal. Behav. 8:e23316.

Ureña-Padilla, A. R., Mackenzie, S. J., Bowen, B. W., and Legard, D. E. 2002 Etiology and population genetics of Colletotrichum spp. causing crown and fruit rot of strawberry. Phytopathology 92:1245-1252.

Ussher, J. R., Elmariah, S., Gerszten, R. E., and Dyck, J. R. 2016. The emerging role of metabolomics in the diagnosis and prognosis of cardiovascular disease. J. Am. Coll. Cardiol. 68:2850-2870.

Vincent, I. M., Creek, D. J., Burgess, K., Woods, D. J., Burchmore, R. J., and Barrett, M. P. 2012. Untargeted metabolomics reveals a lack of synergy between nifurtimox and eflornithine against Trypanosoma brucei. PLoS Negl. Trop. Dis. 6:e1618.

Wastie, R. L. 1972. Secondary leaf fall of Hevea brasiliensis: Factors affecting the production, germination and viability of spores of Colletotrichum gloeosporioides. Ann. Appl. Biol. 72:273-282.

Xu, J., Shao, X., Li, Y., Wei, Y., Xu, F., and Wang, H. 2017. Metabolomic analysis and mode of action of metabolites of tea tree oil involved in the suppression of Botrytis cinerea. Front. Microbiol. 8:1017. 